\title{
Patients Compliance between Hawley Retainers and Vacuum Formed Retainers Following Orthodontic Treatment A Questionnaire Based Study
} Sharath Kumar Shetty ${ }^{1}$, Mahesh Kumar $\mathrm{Y}^{2}$, Neeraj $\mathrm{NS}^{3^{*}}$, Vijayananda K. Madhur ${ }^{4}$

${ }^{1}$ Professor \& HOD, Department of Orthodontics \& Dentofacial Orthopaedics , K.V.G Dental College \& Hospital, Sullia, Karnataka, India

${ }^{2}$ Professor, Department of Orthodontics \& Dentofacial Orthopaedics , K.V.G Dental College \& Hospital, Sullia, Karnataka, India

${ }^{3}$ Post Graduate Student, Department of Orthodontics \& Dentofacial Orthopaedics, K.V.G Dental College \& Hospital, Sullia, Karnataka, India

${ }^{4}$ Reader, Department of Orthodontics \& Dentofacial Orthopaedics , K.V.G Dental College \& Hospital, Sullia, Karnataka, India

DOI: $\underline{10.36347 / \text { sjds.2021.v08i01.004 }}$

| Received: 17.12.2020 | Accepted: 30.12.2020 | Published: 09.01.2021

*Corresponding author: Dr. Neeraj N. S

Abstract

Original Research Article

Retention is considered as an important phase at the end of any active orthodontic tooth movement. Aim: The purposes of this study were to compare compliance between Hawley retainer (HR) and vacuum formed retainers (VFRs) and to detect the reasons for noncompliance. Materials and Methods: Questionnaires were distributed to those who completed orthodontic treatment and currently have experience with the orthodontic retainer. Items included demographic information and questions pertaining to evaluate and quantify retainer compliance during the postretention phase according to several variables, including time in retention, reasons for not wearing etc. Results: Out of the 80 questionnaires distributed, 75 returned and five were excluded from the study. The mean age of the participants in HR group were 23.2( \pm 1.9$)$ years and in VFR group it is $20.2( \pm 1.8)$. Out of the 75 respondents 41 females and 34 males participated in the survey. While comparing the reasons for not using the retainer among the participants, in Hawleys group 39.4\% does not wear due to its aesthetic problems, pain and discomfort, in $10.5 \%$ the retainer didn't fit properly. In VFR group the $59.4 \%$ of the participants not wearing the retainer the reason was found to be the problems associated with speech.in both groups the majority doesn't have any difficulty while removing and wearing the retainer and didn't experienced any wear or breakages during retainer period. Conclusions: Considering all factors related to compliance VFR and HR have equal level of compliance on different factors and the most surveyed reason for not wearing retainer is difficulty in speech and concerns regarding aesthetics.

Keywords: Hawley retainer (HR), vacuum formed retainers (VFRs).

Copyright (C) 2021 The Author(s): This is an open-access article distributed under the terms of the Creative Commons Attribution 4.0 International License (CC BY-NC 4.0) which permits unrestricted use, distribution, and reproduction in any medium for non-commercial use provided the original author and source are credited.

\section{INTRODUCTION}

In 1934, Oppenheim stated the phrase, "Retention is one of the most difficult problems in orthodontia; in fact, it is the problem [1]." Threequarters of a century later that phrase still holds true. Orthodontic literature has been reporting studies on the biological importance of holding teeth in their desired final positions following orthodontic treatment for since the 1950's, yet at present day proper guidelines and protocols for optimal orthodontic retention is still under investigation. This is in part due to the difficulty in controlling and verifying variables such as cooperation, length of retention time, growth, and variations in appliance design [2].

The practice and the theory of orthodontic retention therapy have changed and continue to change over the years. It is currently believed that orthodontic patients should wear their retainers nightly throughout their life after treatment if they desire to maintain optimal dental alignment. Current beliefs are in contrast to the previous position that retainers need not be worn once remodelling of the surrounding periodontium is complete [3].

After orthodontic treatment, there are both a retention phase and a post retention phase of therapy. The retention phase consists of the time during which the periodontium remodels after the skeletal and dental changes associated with orthodontic treatment. The various components that constitute the periodontium complete this process over varying lengths of time it is generally accepted that the retention phase is completed within a year of finishing treatment [4]. The post retention phase of treatment continues for the remainder of the patient's life. During this phase, movement of teeth can occur in response to changing forces in the periodontium caused by continued growth and development. Orthodontic retainers are worn during this phase to offset the effects of these changing forces [3]. 
Many types of removable retainers have been used after active orthodontic treatments to maintain teeth in the final functional and aesthetic position ${ }^{5}$. Hawley retainers (HRs) and vacuum-formed retainers (VFRs) are the most commonly prescribed removable retainers in the orthodontic practice [6]. Many studies have been performed to evaluate the effectiveness of HRs versus VFRs. Most studies have shown no significant difference between both appliances regarding quality of retention [7] except for lateral expansion cases in which HRs are recommended or maxillary/mandibular labial segments' corrections of crowding in which VFRs are recommended [8]. The most disadvantage of using removable retainers is the need of patient's compliance [9]. From reviewing the literature, it can be concluded that discomfort caused by orthodontic appliance wear has a negative influence on the appliance acceptance and on patient's compliance [10-12].

Because most orthodontic retainers are removable, patient compliance is an important factor in almost all cases. Many studies have focused on identifying personal characteristics strongly correlated with a compliant orthodontic patient. However, the data from much of this research have been contradictory, and other studies have yielded inconclusive results [13].

The purposes of this study were to evaluate and quantify retainer compliance during the postretention phase according to several variables, including patient age, sex, time in retention, reasons for not wearing etc.

\section{OBJECTIVES}

The aim of this study was to assess patients' compliance subjectively with vacuum-formed or Hawley retainers after active orthodontic treatment though a questionnaire survey.

\section{Inclusion Criteria}

1. Patients aged 15 to 35 years

2. Both upper and lower dental arches have been orthodontically treated.

3. Full arch orthodontics were performed.

4. The removable appliance has to be worn as retention for post orthodontic treatment.

5. Participants who had either one removable retainer for one arch or two removable retainers for both arches

\section{Exclusion Criteria}

1. Medically compromised patients

2. Patients requiring restorative dental work immediately following orthodontic treatment.

3. Early deboning patients. Those patients who had their braces removed early due to noncompliance, finances, or any other reasons

4. Invisalign Patients

5. Surgical Patients

\section{MATERIALS \& METHODS}

This study was conducted via a questionnaire that was sent through pone to patients who had finished full fixed appliance therapy in the department of orthodontics and dentofacial orthopaedics, KVG dental college $\&$ and hospital sullia. 80 orthodnticaly treated patients who full fill the inclusion and exclusion criteria were enrolled in the study. The surveyed patients included those from both rural and urban environments with various socioeconomic backgrounds. The questionnaires included several identifiers that allowed the respondents to be classified into subgroups. The questionnaire included demographic information and questions pertaining how often he or she was instructed to wear the retainers, how often the retainers were actually worn, and any reasons for not wearing the retainers as instructed the questionnaire is given below. 
The surrey is meant for patients who have received removakle retainers following orthodontic treatment If you have a removable retainer, your comments will provide valuable information for the study. No one will know the identity of the individuals who complete the questionnaire. Consequently, your retextion appointments, or awy further treatment, will not be effected is any way by your response to these questions. By completing and retureing the surrey, you are signing that you understand this study and osesent to your participation in it.

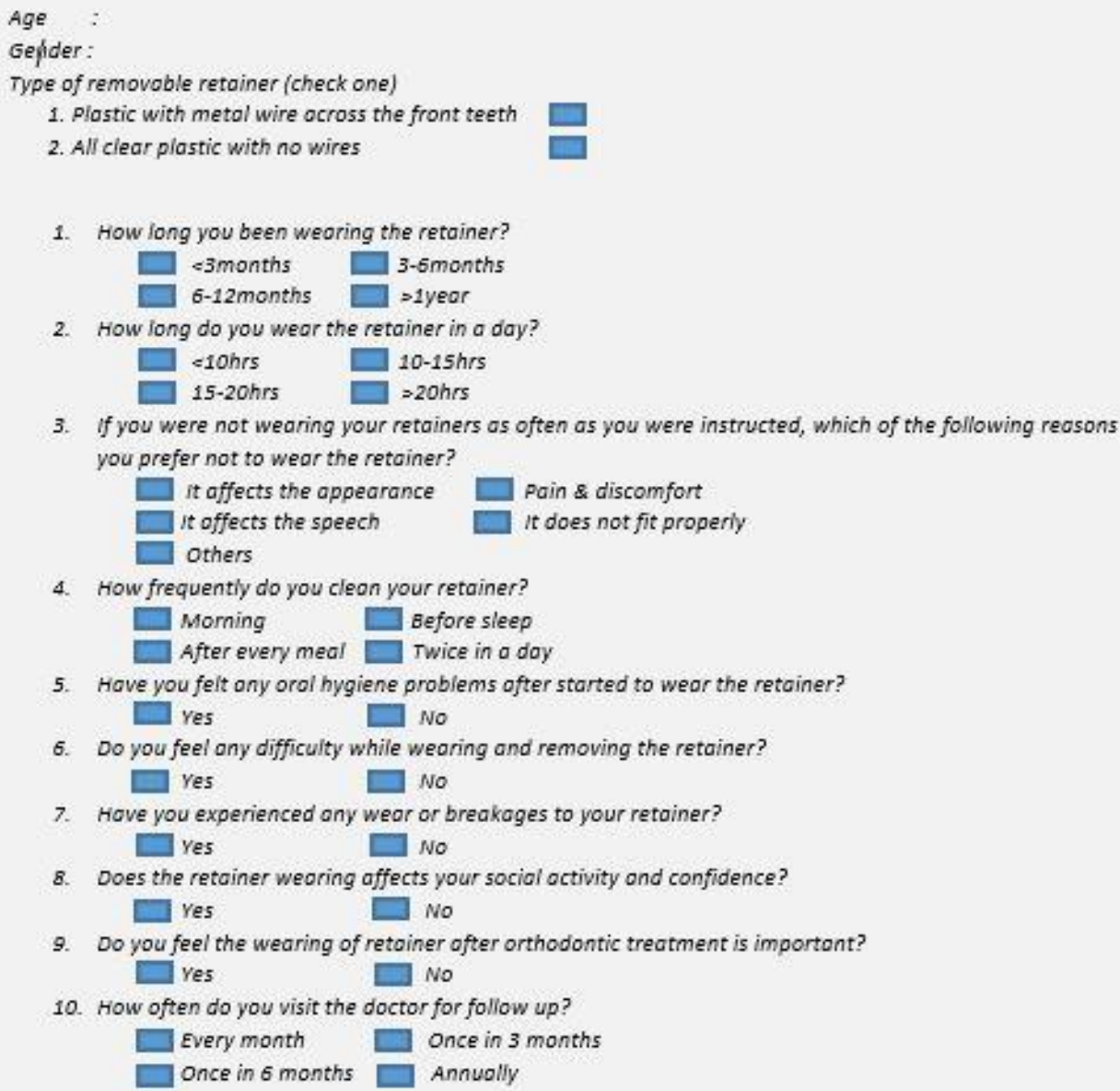

\section{RESULTS}

Out of 80 questionnaires distributed 75 returned with resonses. 38 participants were using Hawleys retainer (HR) and 37 were using vacuum formed retainer (VFR). The mean age of the participants in HR group were $23.2( \pm 1.9)$ years and in VFR group it is $20.2( \pm 1.8)$. Out of the 75 respondents 41 females and 34 males participated in the survey. Demographic characteristics are reported in Table-1. When surveyed about the duration of the wearing retainer $78.94 \%$ of the participants of Hawleys group were wearing for 6-12 months, while VFR group 29.7\% were wearing for $6-12$ months and $35.1 \%$ is wearing for more than 1 year. The duration of participants wearing retainer in a day was seen in both less than 1 year and more than 1 year group. $68.1 \%$ who wore the retainer for more than 1 year were found to be using the retainer for more than $>20 \mathrm{hrs}$ per day. While comparing the reasons for not using the retainer among the participants, in Hawleys group 39.4\% does not wear due to its aesthetic problems, pain and discomfort, in $10.5 \%$ the retainer didn't fit properly. In VFR group the $59.4 \%$ of the participants not wearing the retainer the reason was found to be the problems associated with speech. When compared for frequency of cleaning the retainer more than $65 \%$ of participants in both the groups cleaned the retainer twice a day. When asked about oral hygiene problems due to the retainer use $78.9 \%$ of participants in VFR group and $50 \%$ in HR group had oral hygiene problems. Almost $78 \%$ of participants in both group doesn't have any difficulty while removing and wearing the retainer and didn't experienced any wear or breakages during retainer period. In HR group $89.4 \%$ of participants had issues related to social activity and the wearing affected the confidence while the same problem experienced in VFR group is less than $78.9 \%$. Among the participants more than $60 \%$ doesn't think the retainer wearing is not important after orthodontic treatment. In HR group $60.5 \%$ participants visit their dentist for follow up once in 3 months while $89.1 \%$ of participants in VFR group visits dentists only annually. While assessing the compliance factors like reasons for not wearing the retainer, oral hygiene problems associated while using retainers, regular follow up to the dentists, and the importance for wearing retainer after orthodontic treatment was found to be statistical significant. 
Table-1: Demographic characteristics

\begin{tabular}{|l|l|l|l|l|}
\hline & N & Hawleys retainer group & Vacuum formed retainer group & P value \\
\hline Age & 75 & $23.2 \pm 1.9$ & $20.2 \pm 1.8$ & 0.43 \\
\hline Gender & & & \multirow{2}{*}{0.22} \\
\hline Male & 34 & $19(55 \%)$ & $15(44.2 \%)$ & \\
\hline Female & 41 & $20(48.7 \%)$ & $21(51.3 \%)$ & \\
\hline
\end{tabular}

\begin{tabular}{|c|c|c|c|}
\hline & Hawleys retainer group & Vacuum formed retainer group & \multirow[t]{2}{*}{$P$ value } \\
\hline $\mathrm{N}$ & 38 & 37 & \\
\hline \multicolumn{4}{|c|}{ Duration of wearing retainer } \\
\hline$<3$ months & $1(2.6 \%)$ & $3(8.1 \%)$ & \multirow{4}{*}{0.6} \\
\hline 3-6 months & $4(10.5 \%)$ & $10(27 \%)$ & \\
\hline 6-12 months & $30(78.94 \%)$ & $11(29.7 \%)$ & \\
\hline$>1$ year & $3(7.8 \%)$ & $13(35.1 \%$ & \\
\hline \multicolumn{4}{|c|}{ Reason for not wearing retainer } \\
\hline Appearance & $15(39.4 \%)$ & $2(5.4 \%)$ & \multirow{5}{*}{$0.03 *$} \\
\hline Pain & $15(39.4 \%)$ & $6(16.2 \%)$ & \\
\hline Speech & $2(5.2 \%)$ & $22(59.4 \%)$ & \\
\hline Fit & $4(10.5 \%)$ & $3(8.1 \%)$ & \\
\hline Others & $2(5.5 \%)$ & $4(10.9 \%)$ & \\
\hline \multicolumn{4}{|c|}{ Frequency of cleaning retainer } \\
\hline Morning & $5(13.1 \%)$ & $6(16.2 \%)$ & \multirow{4}{*}{0.9} \\
\hline Before sleep & $4(10.5 \%)$ & $4(10.8 \%)$ & \\
\hline After meal & $2(5.2 \%)$ & $2(5.4 \%)$ & \\
\hline Twice in a day & $27(70.05 \%)$ & $25(67.5 \%)$ & \\
\hline \multicolumn{4}{|c|}{ Visiting the doctor for follow-up } \\
\hline Every month & $3(7.8 \%)$ & $1(2.7 \%)$ & \multirow{4}{*}{$0.04 *$} \\
\hline Once in 3 months & $23(60.5 \%)$ & $1(2.7 \%)$ & \\
\hline Once in 6 months & $8(21 \%)$ & $2(5.4 \%)$ & \\
\hline annually & $4(10.5 \%)$ & $33(89.1 \%)$ & \\
\hline
\end{tabular}

\begin{tabular}{|l|l|l|l|}
\hline & $\begin{array}{l}\text { Hawleys retainer } \\
\text { group }\end{array}$ & $\begin{array}{l}\text { Vaccum formed } \\
\text { retainer group }\end{array}$ & $\begin{array}{l}\text { P } \\
\text { value }\end{array}$ \\
\hline $\mathrm{N}$ & 38 & 37 & $0.01 *$ \\
\hline Oral hygiene problems & $19(50 \%)$ & $30(78.9 \%)$ & 0.5 \\
\hline Difficulty in use & $8(21 \%)$ & $8(21 \%)$ & 0.7 \\
\hline Wear or breakage & $30(78.94 \%)$ & $30(78.9 \%)$ & 0.9 \\
\hline Social activity and confidence & $34(89.4 \%)$ & $30(78.9 \%)$ & $0.02 *$ \\
\hline $\begin{array}{l}\text { Importance of wearing orthodontic } \\
\text { retainer after treatment }\end{array}$ & $23(60.5 \%)$ & $25(67.5 \%)$ & \\
\hline
\end{tabular}

\begin{tabular}{|c|c|c|c|}
\hline & \multicolumn{2}{|c|}{ Years of using retainer } & \multirow{3}{*}{$\mathrm{P}$ value } \\
\hline & $<1$ year & $>1$ year & \\
\hline $\mathrm{N}$ & 53 & 22 & \\
\hline \multicolumn{4}{|c|}{ Duration of wearing retainer in a day } \\
\hline$<10$ hours & $1(1.8 \%)$ & $1(4.5 \%)$ & \multirow[t]{4}{*}{$0.012 *$} \\
\hline $10-15$ hours & $17(32 \%)$ & $2(9 \%)$ & \\
\hline $15-20$ hours & $31(58.4 \%)$ & $4(18.1 \%)$ & \\
\hline$>20$ hours & $4(7.5 \%)$ & $15(68.1 \%)$ & \\
\hline
\end{tabular}

\section{DISCUSSION}

The significant higher acceptance levels of appearance in the VFR group can be explained by the clear material of the VFR and the less visibility compared to the visible metallic labial bow of the HR. The labial wire of the HRs was visible, and a number of subjects expressed mild dissatisfaction. This agrees with the results of Hichens et al., [14]. On the other hand, Pratt et al., found no differences regarding aesthetic aspects between the VFR and the HR group [15].

One possible explanation for the faster decrease in retainer compliance with VFRs is differences in durability between the 2 retainer types. Because VFRs cover the occlusal surfaces, they tend to 
break down under the stresses of functional and parafunctional activities. For the most part, Hawley retainers do not cover the occlusal surfaces and are therefore less vulnerable to wear over time. Another factor that might influence compliance is the increased time required to maintain and clean a VFR. The wear and the flexibility of the VFR make it more susceptible to fractures, stains, and absorption of oral fluids [9].

The significant higher levels of comfort with the VFR may be due to the higher levels of appearance acceptance, oral hygiene perception, swallowing ability, talking ability, and self-confidence with significant less level of gingival irritation. The lighter weight of VFR may be an additional reason although it was not measured in the current study. Wong and Freer conducted survey research in 2006 that found a strong relationship between compliance with removable retainers and patient's perception on its comfort. Hichens et al discovered through a patient satisfaction questionnaire that most people preferred the vacuumformed retainer over Hawley retainers. Mollov et al., reported in a survey study including mostly college students and dental students similar increase in patient satisfaction with VFR's as compared to Hawleys [16].

Higher significant levels of self-confidence were also assessed in this study in subjects of VFR group. The significant higher levels of self-confidence with the VFR may be related to its clear appearance with less visibility and higher ability to talk when the retainer is in situ This is consistent with the findings of Hichens et al., who found that using HRs caused more embarrassment than using VFRs [17].

The significant higher levels of perception of good hygiene with the VFR group can be a result of the apparently higher levels of visibility of food remnants beneath the appliances compared to the HRs. The significant less levels of gingival irritation with the VFRs can be attributed to the absence of retentive metal elements such as Adams clasps and the labial bow.

Due to their form, vacuum-formed thermoplastic retainers may have an increased accumulation of material inside their concavities and greater difficulty in cleaning the retainer with the toothbrush. In addition, the higher incidences of little and serious breakages of the vacuum-formed thermoplastic Essix retainers may induce the patient to perform a less vigorous cleaning of the retainer, with consequent plaque and calculus accumulations and gingival problems over time. Gingival problems were also confirmed by the higher percentage of bleeding sites in the Essix group $(30.35 \%)$ compared with the Hawley group (11.30\%) [18].

One issue that was addressed in this study was the frequency of follow-up visits. After deboning and retainer delivery patients were instructed to visit back to the department for the regular follow up visits once in every month After the first year, retainer checks typically occur Very often. However, not all patients return for their retainer-check appointments. Retainer checks have several benefits, including reinforcing proper retainer wear, examining retainers for breakages and wear, evaluating the fit of the retainers, and identifying any other problems related to retention. In this study, we identified that in HR group 55\% had regular monthly visit while it is $32 \%$ in VFR group. It is likely that those who returned for these follow-up visits would be more likely to have proper retainer compliance [19].

Another factor that might affect our results was the low percentage of nonrespondents in this study. This include less than 1\% in both Hawley's and vacuum formed retainer group. Although this model is a good indicator of how the above factors impact patient compliance with retainers, the model would most likely better represent the general population if there had been full respondents. It is reasonable to assume that non respondents were less compliant on average with orthodontic retainers than those who did respond.

The results of this study also suggest that, although compliance is better with HRs than with VFRs, overall patient compliance with removable retainers is not acceptable as many participants which include $35 \%$ of HR and $25 \%$ of VFR who reported that they are not using retainers due to different above mentioned reasons or they had lost their retainer. Because of this fixed retention should be evaluated as a potentially preferred alternative to removable retainers [19].

\section{CONCLUSIONS}

Considering all factors related to compliance VFR and HR have equal level of compliance on different factors and the most surveyed reason for not wearing retainer is difficulty in speech and concerns regarding aesthetics. Among the participants majority had an opinion that retainer wearing is not important after orthodontic treatment hence, regular follow up by an orthodontist and motivation on wearing retainer will make much difference.

\section{REFERENCES}

1. Oppenheim A. The crisis in orthodontia. I. Tissue changes during retention: Skogsborg's septotomy. Int J Orthod Dent Child. 1934; 20:640.

2. Reitan K. Tissue rearrangement during retention of orthodontically rotated teeth. Angle Orthod. 1958; 29:105-13.

3. Thilander B. Orthodontic relapse versus natural development. Am J Orthod Dentofacial Orthop. 2000; 117:562-3.

4. Melrose C, Millett DT. Toward a perspective on orthodontic retention? Am J Orthod Dentofacial Orthop. 1998; 113:507-14. 
Sharath Kumar Shetty et al., Sch J Dent Sci, Jan, 2021; 8(1): 19-24

5. Graber TM, Vanarsdall RL, Vig KWL. Orthodontics: Current Principles and Techniques. Elsevier/Mosby, St. Louis, MO. 2005.

6. Mai W, He J, Meng H, Jiang Y, Huang C, Li M, Yuan K, Kang N. Comparison of vacuum-formed and Hawley retainers: a systematic review. American Journal of Orthodontics and Dentofacial Orthopedics. 2014; 145:720-727.

7. Lindauer SJ, Shoff, RC. Comparison of Essix and Hawley retainers. Journal of Clinical Orthodontics, 1998; 32:95-97.

8. Rowland H, Hichens L, Williams A, Hills D, Killingback N, Ewings P, Clark S, Ireland AJ, Sandy JR. The effectiveness of Hawley and vacuum-formed retainers: a single-center randomized controlled trial. American Journal of Orthodontics and Dentofacial Orthopedics. 2007 Dec 1;132(6):730-7.

9. Ledvinka J. Vacuum-formed retainers more effective than Hawley retainers. Evidence Based Dentistry. 2009; 10:47.

10. Hyun P, Preston CB, Al-Jewair TS, Park-Hyun E, Tabbaa S. Patient compliance with Hawley retainers fitted with the SMART(R) sensor: a prospective clinical pilot study. Angle Orthodontist. 2015; 85:263-269.

11. Egolf RJ, BeGole EA, Upshaw HS. Factors associated with orthodontic patient compliance with intraoral elastic and headgear wear. American Journal of Orthodontics and Dentofacial Orthopedics, 1990; 97:336-348.

12. Nanda RS, Kierl MJ. Prediction of cooperation in orthodontic treatment. Am J Orthod Dentofacial Orthop. 1992; 102:15-21.
13. Ireland A, Sandy J. Cost-effectiveness and patient satisfaction: Hawley and vacuum-formed retainers. European Journal of Orthodontics, 2007; 29:372-378.

14. Pratt MC, Kluemper, GT, Lindstrom AF. Patient compliance with orthodontic retainers in the postretention phase. American Journal of Orthodontic and Dentofacial Orthopedics, 2011; 140:196-201.

15. Mollov ND, Lindauer SJ, Best AM, Shroff B, Tufekci E. Patient attitudes toward retention and perceptions of treatment success. Angles Orthod. 2010; 80:656-661.

16. Hichens L, Rowland H, Williams A, Hollinghurst S, Ewings P, Clark S, Ireland A, Sandy J. Costeffectiveness and patient satisfaction: Hawley and vacuum-formed retainers. European Journal of Orthodontics. 2007; 29:372-378.

17. Gardner GD, Dunn WJ, Taloumis L. Wear comparison of thermoplastic materials used for orthodontic retainers. Am J Orthod Dentofacial Orthop. 2003; 124:294-7.

18. Pratt MC, Kluemper GT, Lindstrom AF. Patient compliance with orthodontic retainers in the postretention phase. Am J Orthod Dentofacial Orthop. 2011; 140:196- 201.

19. Hichens L, Rowland H, Williams A, Hollinghurst S, Ewings P, Clark S, Ireland A, Sandy J. Costeffectiveness and patient satisfaction: Hawley and vacuum-formed retainers. The European Journal of Orthodontics. 2007 Aug 1;29(4):372-8. 